\title{
Physiologically driven avian vocal synthesizer
}

\author{
Jacobo D. Sitt, ${ }^{1}$ Ezequiel M. Arneodo, ${ }^{1}$ Franz Goller, ${ }^{2}$ and Gabriel B. Mindlin ${ }^{1}$ \\ ${ }^{1}$ Departamento de Física, FCEN, Universidad de Buenos Aires, Ciudad Universitaria, Pab. I, 1428 Buenos Aires, Argentina \\ ${ }^{2}$ Department of Biology, University of Utah, Salt Lake City, Utah, 84112 USA
}

(Received 7 December 2009; revised manuscript received 1 February 2010; published 31 March 2010; publisher error corrected 6 April 2010)

\begin{abstract}
In this work, we build an electronic syrinx, i.e., a programmable electronic device capable of integrating biomechanical model equations for the avian vocal organ in order to synthesize song. This vocal prosthesis is controlled by the bird's neural instructions to respiratory and the syringeal motor systems, thus opening great potential for studying motor control and its modification by sensory feedback mechanisms. Furthermore, a well-functioning subject-controlled vocal prosthesis can lay the foundation for similar devices in humans and thus provide directly health-related data and procedures.
\end{abstract}

DOI: 10.1103/PhysRevE.81.031927

Complex motor behavior emerges from the interactions between a nervous system and peripheral effectors systems [1]. This interplay is clearly illustrated in birdsong production, where a highly nonlinear device is capable of generating a variety of acoustically different sounds, even when driven by relatively simple physiological instructions $[2,3]$. Recently, the modeling of the avian vocal organ has helped to understand the relationships between different acoustic features, which are not under direct neural control but are determined by the biomechanics of the peripheral system $[2,3]$. In this work, we implement an electronic device which continuously reads physiological instructions driving the syrinx and integrates the model equations ruling its dynamics in the time lapse between readings.

The most widely studied songbird species is the zebra finch (Taeniopygia guttata), whose song consists of three to eight distinct song syllables with a variety of acoustic characteristics. Whereas many songbird species produce sounds with low upper harmonic content (tonal), zebra finch song is composed of both spectrally rich and tonal syllables. In a previous work, we found that there is a relationship between the spectral content of a vocalization and its fundamental frequency [2]. Moreover, this relationship can be explained in terms of the different dynamical mechanisms by which labial oscillations are started when the air sac pressure reaches a threshold value [Hopf mechanism versus a saddle node in a limit cycle (SNILC) bifurcation; e.g., [4]]. These different mechanisms were found in a physical model for birdsong production $[2,3]$, which illustrates that some acoustic features of the song are not under direct control of the nervous system but emerge from the interactions with the biomechanical device.

One of the first low-dimensional models for the dynamics of a membrane in an airflow was proposed by Titze and Martin [5] and has been used to describe the source in birdsong [6]. The model assumes that for high-enough values of the airflow, soft pieces of tissue (labia, in the case of birds) start to oscillate. The modulations of the airflow are the responsible for the sound. The motion of the oscillating tissues, in this model, is represented as a surface wave propagating in the direction of the airflow. In order to describe this wave, Titze assumed two basic modes: a lateral displacement of the tissues and a flappinglike motion responsible for an out-of-
PACS number(s): 87.19.1u, 87.10.Ed

phase oscillation of the top and bottom parts of the membranes.

It is possible to coordinate these modes in such a way that the system gains energy in each cycle. It suffices that the labia present a convergent profile when they move away from each other and a divergent (or less convergent) one when they approach. In this way, there will be higher interlabial pressure when the labia are moving apart than when they are approaching, since a convergent profile guarantees an interlabial pressure similar to the (high) subglottal pressure.

A kinematic description of this scenario (in which the movement is ultimately an upward propagating wave on a labium) can be carried out in terms of $a_{1}$ and $a_{2}$, the half separation between the lower and upper edges of the labia. Under the hypothesis of a wavelike motion, these half separations can be written in terms of the midpoint position of a membrane, $x$, and its velocity, $y$. If the time that it takes the wave to propagate half the vertical size of the labia is $\tau$, the half separations between the edges of the membranes will satisfy

$$
\begin{aligned}
& a_{1}=a_{01}+x+\tau y, \\
& a_{2}=a_{02}+x-\tau y,
\end{aligned}
$$

where $a_{01}$ and $a_{02}$ are the half separations at the rest state. The average pressure $p_{a v}$ between the labia [5] is then

$$
p_{a v}=p_{s}\left(1-\frac{a_{2}}{a_{1}}\right),
$$

where $p_{s}$ stands for the sublabial pressure. With these elements, we can write the dynamical equations for $x$, taking into account dissipation, elastic restitution, and nonlinear dissipation

$$
\begin{gathered}
\frac{d x}{d t}=y \\
\frac{d y}{d t}=(1 / m)\left[-k(x) x-b(y) y-c x^{2} y+a_{l a b} p_{s}\left(\frac{\Delta a+2 \tau \tau}{a_{01}+x+\tau y}\right)\right] .
\end{gathered}
$$

The first term corresponds to a nonlinear restitution force, where $k(x)=k_{1}+k_{2} x^{2}$. The second term accounts for dissipa- 
(a)
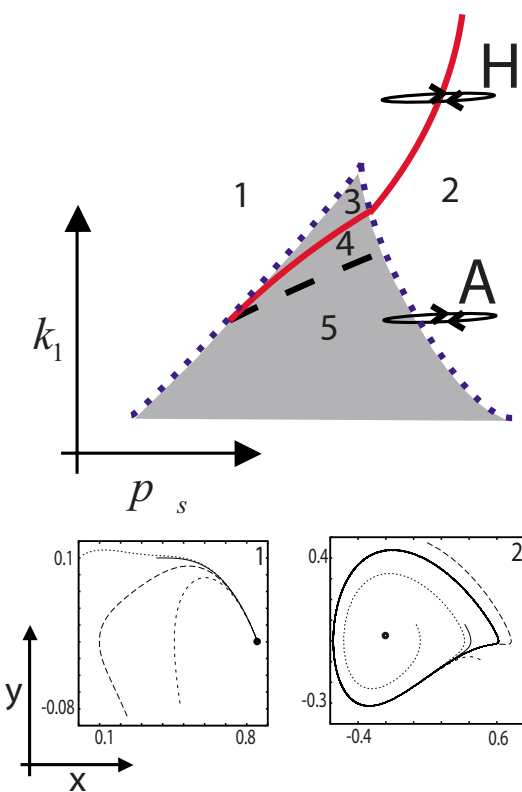

(b)
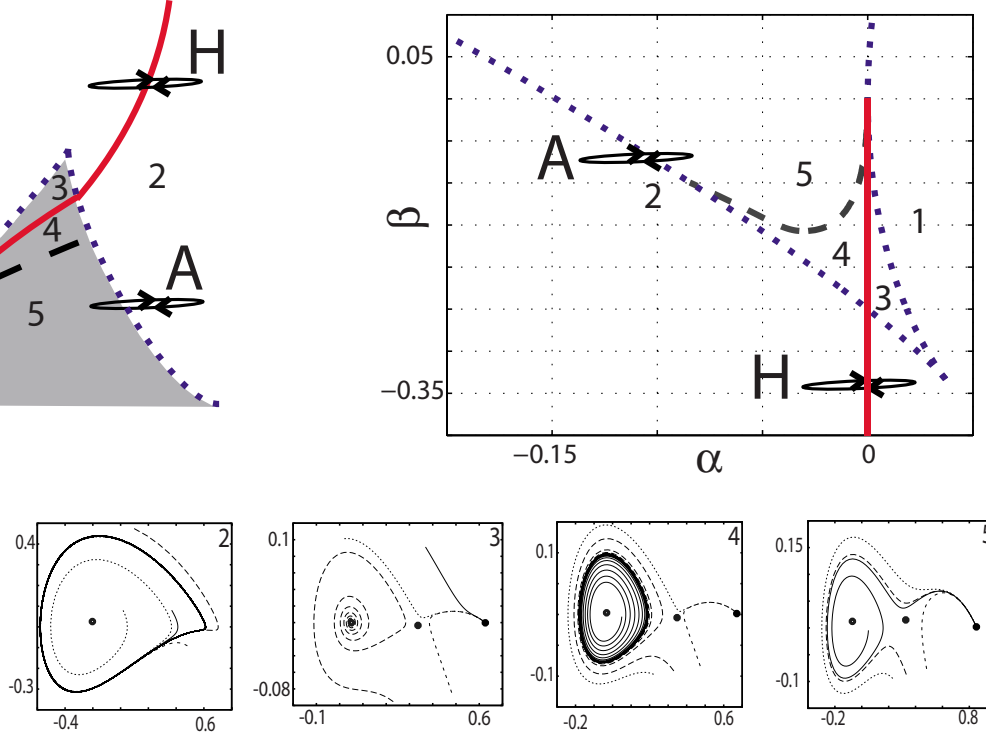
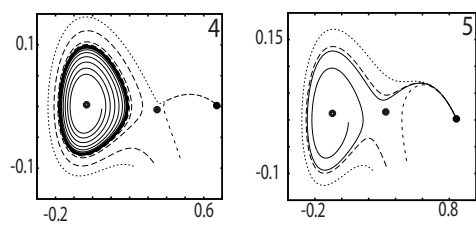

FIG. 1. (Color online) Bifurcation diagram for (a) the physical and (b) the normal form models. For the physical model, the diagram is displayed in the air sac pressure and the tension parameter space $\left(p_{s}, k_{1}\right)$ and for the normal form model in the $(\alpha, \beta)$ parameter space. The existence of neighboring regions as 1 and 2, and regions 5 and 2 is the mathematical condition for a model to be capable of displaying both tonal and spectrally rich sounds with well-defined fundamental frequencies. Typical portraits of the $(x, y)$ phase spaces for each region are shown in the corresponding lower insets. In both upper panels, continuous thick line represents the Hopf bifurcation, dashed line the homoclinic bifurcation, and dotted blue lines the saddle-node bifurcations. Paths A and $\mathrm{H}$ represent the different ways to start oscillation of the labia by crossing the Hopf $(\mathrm{H})$ and the SNILC (A) bifurcation lines, thus generating respectably either tonal or spectrally rich sounds.

tion, with $\beta(y)=\beta_{1}+\beta_{2} y^{2}$. The third term is also a nonlinear dissipation that becomes relevant as $x$ takes large values, corresponding to large departures from the rest position. In this way, this position-dependent nonlinear dissipation serves to model collisions between labia or with containing walls, either one bounding their motions. Finally, the last term describes the effect of the interlabial pressure and $\Delta a=a_{01}$ $-a_{02}$

It is interesting to notice that the existence of nonlinear components in the restitution forces for the labia was recently found to play an important role in the generation of low-frequency oscillations of rich spectral content. In previous work [3], it was shown that both Hopf and SNILC bifurcations could occur. In the first case, harmonic oscillations are born with finite frequency and zero amplitude. In the latter, a saddle and a node collide, in a way such that the unstable manifold of the saddle belongs to the stable manifold of the node. In this way, an oscillation is born with zero frequency and nonzero amplitude. These oscillations were found to be consistent with spectrally rich sounds in the zebra finch $[2,3]$.

The bifurcation diagram, in terms of $p_{s}, k_{1}$, is displayed in Fig. 1(a) (adapted from [3]). In the shaded region, three fixed points exist. The curves that define its border correspond to saddle-node bifurcations, where a pair of fixed points, one stable and one unstable, collides. The solid, thick lines represent Hopf bifurcation curves, where fixed points lose stability against periodic solutions. The point at which a Hopf curve meets tangentially a saddle-node curve is a TakensBogdanov bifurcation point: a linear singularity with two zero eigenvalues. The complete set of qualitatively different solutions found in its vicinity is displayed in the figure.

A thorough analysis of the solutions of the physical model that we proposed for the production of zebra finch song [2] can be found in [3]. The generation of syllables with different spectral contents occurs, according to the model, when the bird modulates the physiological instructions in order to follow paths as the ones indicated in the figure. The path for high values of $k_{1}$ (regions 1 and 2) generates tonal sounds, whereas the path for low $k_{1}$ values gives rise to harmonic stacks (regions 2-5).

Since we are interested in carrying out an integration of our model in real time with a digital signal processor (DSP), we found it convenient to find an equivalent set of equations which would imply computationally less expensive operations. By equivalent we mean a set of equations that is capable of displaying topologically equivalent sets of solutions as the parameters are varied. In particular, it was desirable to find a system of equations not involving a ratio of polynomial functions of the variables.

This is a standard problem in dynamical systems: to find the simplest vector field presenting topologically equivalent solutions as those of a given problem. This procedure is algorithmic: at a linear singularity, it is possible to find nonlinear changes of variables eliminating the nonresonant terms in a power-series expansion of the vector field.

Let us start our simplifying procedure where the system presents a Takens-Bogdanov singularity. Then, the vector field, at third order, can be written in its normal form as [7] 


$$
\begin{gathered}
\frac{d x}{d t}=y, \\
\frac{d y}{d t}=c_{11} x^{2}+c_{12} x y+c_{21} x^{3}+c_{22} x^{2} y .
\end{gathered}
$$

By performing a normal form reduction, we can find a nonlinear change of coordinates to eliminate the nonresonant terms. The fixed point then reads

$$
x=-\frac{k_{1}}{3 k_{2} a_{01}}-\sqrt{\left(\frac{k_{1}}{3 k_{2} a_{01}}\right)^{2}-\frac{k_{1}}{3 k_{2} a_{01}}} .
$$

Performing a Taylor expansion around the fixed point at the parameters $p_{s}^{*}$ and $k_{1}^{*}$ (chosen numerically in such a way that both the saddle node and the Hopf conditions are satisfied), we get

$$
\begin{gathered}
\frac{d \varepsilon_{x}}{d t}=\varepsilon_{y}, \\
\frac{d \varepsilon_{y}}{d t}=\left(-3 k_{2} x+\frac{p_{s}^{*} \Delta a}{\left(a_{01}+x\right)^{3}}\right) \varepsilon_{x} \\
-\left(2 \beta_{2} x+\frac{2 \tau \tau_{s} \Delta a}{\left(a_{01}+x\right)^{3}}+\frac{2 \tau \tau_{s}}{\left(a_{01}+x\right)^{3}}\right) \varepsilon_{x} \varepsilon_{y}+\cdots,
\end{gathered}
$$

where $\varepsilon_{y}=y$ and $\varepsilon_{y}=x-x^{*}$. Evaluating these coefficients at the chosen parameter values, we find that $c_{11}>0 ; c_{12}<0$. As it is the standard procedure in the reduction to the standard form of the Takens-Bogdanov bifurcation, a nonlinear change of variables allows us to get rid of the term in $\varepsilon_{y}^{2}$. This change of variables will affect the cubic coefficients obtained in the expansion. Yet, we chose cubic phenomenological saturation terms (and therefore, after the normal form reduction, present components in $x^{3}$ and $x^{2} y$ ) and therefore $c_{21}$ and $c_{22}$ will be negative. In this way, after scaling the variables, at the parameters of the Takens-Bogdanov bifurcation, our system will be equivalent to

$$
\begin{gathered}
\frac{d x}{d t}=y, \\
\frac{d y}{d t}=x^{2}-x y-x^{3}-x^{2} y .
\end{gathered}
$$

We performed an unfolding of our vector field as the one proposed by Takens and the resulting bifurcation diagram is shown in Fig. 1(b). It presents the same set of dynamical scenarios, but the vector field only involves polynomial nonlinear terms, making it suitable for a digital implementation by a DSP. In other words, we obtained the simplest mathematical model (i.e., with the smallest number of nonlinear terms) that satisfies the conditions required for the generation of both tonal and spectrally rich sounds with well-defined fundamental frequencies, i.e., which presents an equivalent bifurcation diagram as our physical model [see Fig. 1(b)].

Since this reduced model contains a minimal number of nonlinear terms, it saves computational time without losing the required dynamical properties, keeping the parameters

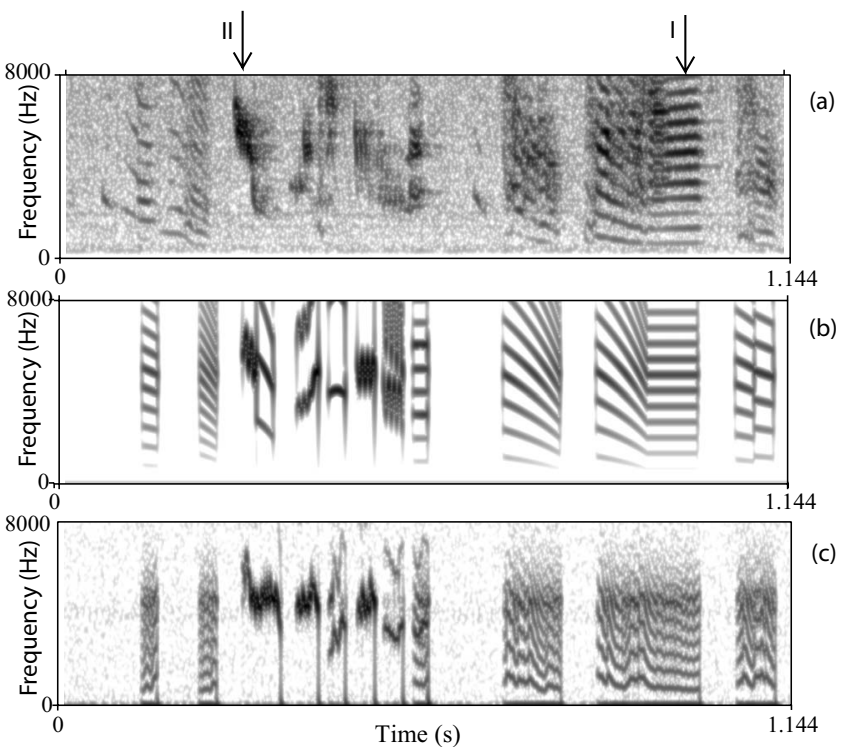

FIG. 2. (Upper panel) Natural song. (Middle panel) Synthetic song generated by the computational model with ad hoc parameters. (Bottom panel) Physiologically driven electronic syrinx implemented in a DSP. $L=1.9 \mathrm{~cm}, r=-0.9$. Arrows indicate the segments used to fit.

within a physiologically meaningful range. We introduced a $\gamma$ time scaling factor in order to match the velocity of the oscillations in the model variables and the measured sound pressure fluctuations, which are assumed to be caused by labial modulation of the airflow; i.e., with this change of variables $(t \rightarrow t / \gamma, y \rightarrow \gamma y)$, the final model reads

$$
\begin{gathered}
\frac{d x}{d t}=y, \\
\frac{d y}{d t}=\gamma^{2} \alpha+\gamma^{2} \beta x+\gamma^{2} x^{2}-\gamma x y-\gamma x^{3}-\gamma x^{2} y,
\end{gathered}
$$

where $x$ represents the departure of the midpoint position of the oscillating labia in the syrinx (which are the tissues modulating airflow to produce sound), $\gamma$ is a time scaling factor, and the parameters $\alpha$ and $\beta$ are functions of the air sac pressure and the activity of the ventral syringeal muscle, respectively. These functions are defined by the transformation of variables taking the physical model to the normal form as described before. Synthetic sound can be generated with this model if the sound pressure at the input of the tract is computed as $P_{i}(t)=x(t)-r P_{i}(t-2 L / v)$ [8,9], where $L$ stands for the length of a tube representing the tract, $v$ is the sound velocity, and $r$ the reflection coefficient of the sound wave. The output pressure of the vocal tract, which reads $P_{t}(t)=(1-r) P_{i}(t-2 L / v)$, is computed as the output sound. This expression reflects that the output pressure is the transmitted sound wave at the end of the tract.

In order to fit the parameters in our model, we chose two segments of the song whose sonogram is displayed in Fig. 2 (upper panel). One segment corresponds to a high-frequency, tonal sound, while the other to a low-frequency, spectrally rich sound. We computed fundamental frequencies and spec- 
tral contents of those two time series segments. We defined pitch $\left(f_{0}\right)$ as fundamental frequency and spectral content index (SCI) as described in [2]: an index $(S)$ quantifying how much energy is distributed in the multiples of the fundamental frequency. In order to fit $\gamma$, we computed these values for the first segment $\left(f_{0}=715 \mathrm{~Hz}, S=6.6\right)$. Then, we integrated the equations for different values of $\gamma$ and $\beta$. For each $\gamma$, we looked for the value of $\beta$ to give rise to the same oscillation frequency as our experimental segment and the synthetic SCI was computed. The distance $d_{1}$ between the recorded and synthetic values, $d_{1}=\left(\frac{S_{1, s}-S_{1, r}}{S_{1, s}}\right)^{2}$, where $S_{1, s}$ represents the SCI for the synthetic sound and $S_{1, r}$ for the recorded one, was calculated as a function of $\gamma$. The same procedure was followed for the second segment $\left(f_{0}=5468 \mathrm{~Hz}, S=1.0245\right)$ to compute distance $d_{2}$. The quantity $d_{\text {total }} \equiv d_{1}+d_{2}$ was then calculated as a function of $\gamma$. We found a minimum for $\gamma$ $=23500$.

In order to produce parameter paths capable of driving the model to generate synthetic sound similar to the recorded one, we defined two regimes. High-frequency sounds (sounds with fundamental frequencies above $1.8 \mathrm{kHz}$ ) were assumed to be produced when labial oscillations were turned on in Hopf bifurcations; a dynamical scenario compatible with tonal oscillations [2]. Low-frequency sounds (fundamental frequencies smaller than $1.2 \mathrm{kHz}$ ) were assumed to be uttered when labial oscillations were born in SNILC bifurcations [2]. In the cases where no spectral discontinuities were observed in the low-frequency syllables, both sources were assumed to be active simultaneously and operating at the same dynamical regime. We built two time series $\beta^{\text {numeric }_{1,2}(t) \text {, each corresponding to either of the mentioned }}$ regimes. Each time series $\beta_{\text {numeric }_{1,2}(t)}$ was built in such way that the fundamental frequency of the synthesized song matches the fundamental frequency of the uttered song at that time. Each $\beta^{\text {numeric }_{1,2}(t)}$ was used to drive the model, independently, to produce two series of pressure fluctuations that were added to emulate the pressure at the input of the vocal tract in order to produce synthetic song. The parameter $\alpha$ was chosen so that the variable $x$ would oscillate when phonation was detected. We chose $\alpha$ to take either $\alpha_{\text {off }}$ $=0.05$ or $\alpha_{\text {on }}=-0.15$ depending on whether the oscillations are off or on, respectively. Notice that a low-frequency harmonic down sweep in the syllable and a brief down sweep around $t=0.66 \mathrm{~s}$ are not modeled. Published results [11] suggest that those sounds might have their dynamical origin in nonlinear effects due to source-source interactions, not included in the modeling here presented.

In order to quantify the similarity between the natural and the synthesized songs, we measured pitch and spectral content in each of the recordings. Pitch was extracted using fixed $10 \mathrm{~ms}$ time steps, obtaining pitch time series for each of the recordings. Mean pitch distance was defined as

$$
\left\langle d_{\text {pitch }}\right\rangle=\frac{\sum_{i=1}^{n}\left|f_{01}(i)-f_{02}(i)\right|}{\sum_{i=1}^{n} f_{01}(i)},
$$

with $f_{01}$ corresponding to the pitch of the experimental recordings and $f_{02}$ the pitch of synthesized song. Mean dis-

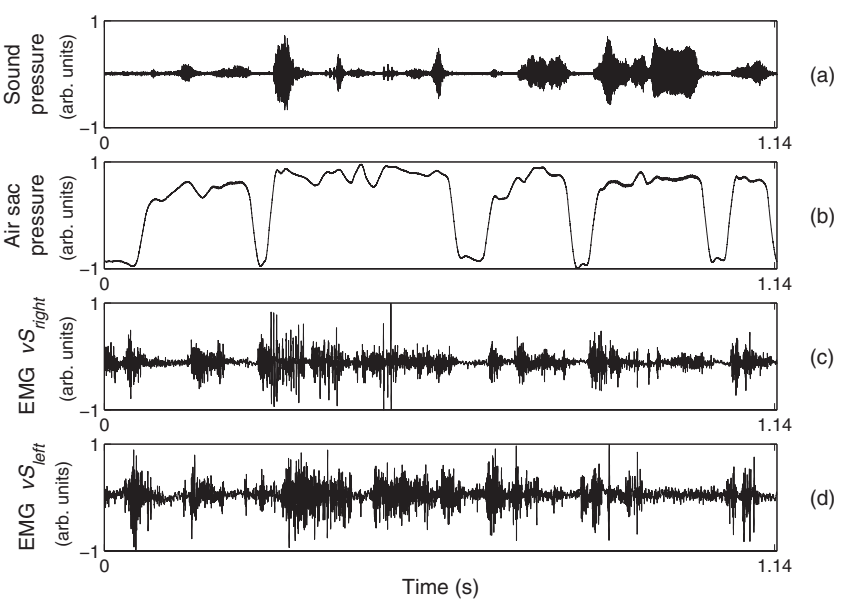

FIG. 3. Simultaneous experimental recordings while the bird was singing of (a) sound pressure, (b) air sac pressure, (c) right $v S$ EMG, and (d) left $v S$ EMG.

tance between experimental and synthetic series was $\left\langle d_{\text {pitch }}\right\rangle$ $=0.152$ with $\mathrm{STD}=0.262$. We calculated SCI in a time frame of $2.7 \mathrm{~ms}$ surrounding each of the time steps where pitch was measured. The mean spectral content distance $d_{\mathrm{SCI}}$ is defined as

$$
\left\langle d_{\mathrm{SCI}}\right\rangle=\frac{\sum_{i=1}^{n}\left|S_{1}(i)-S_{2}(i)\right|}{\sum_{i=1}^{n} S_{1}(i)},
$$

where $S_{1}$ is the natural song SCI series and $S_{2}$ the synthetic one. Mean distance between natural and synthetic series was $\left\langle d_{\mathrm{SCI}}\right\rangle=0.263$ with $\mathrm{STD}=0.262$. The resulting synthetic song generated by driving the equations with $\beta^{\text {numeric }_{1,2}(t)}$ is displayed in the middle panel of Fig. 2.

Once identified the range of time-dependent parameters that lead to synthetic song with similar fundamental frequency and spectral content behavior, we proceeded to (1) find a transformation capable of mapping actual electromyographic (EMG) data from a singing bird to parameters within the range computed above and (2) implement this model into a DSP.

Properly scaling EMG signals from syringeal muscles within the range of the previously computed time-dependent parameters allows synthesizing realistic song. In order to record muscle activities, wire electrodes were implanted into syringeal muscles. The electrodes were prepared from insulated stainless-steel wire and secured to the tissue with a microdrop of tissue adhesive. Before closing the air sac, all the wires were led out and routed to the back. Simultaneous recordings of sound, pressure, right $v S$ EMG activity, and left $v S$ EMG activity are shown on Fig. 3. The rectified, smoothed envelope of the EMG provided a relative measure of the muscle activity [9]. This was used to understand how muscles contribute to the movements responsible for the slow evolution of the fundamental frequency within song syllables [10]. This preprocessing of the EMG data was per- 


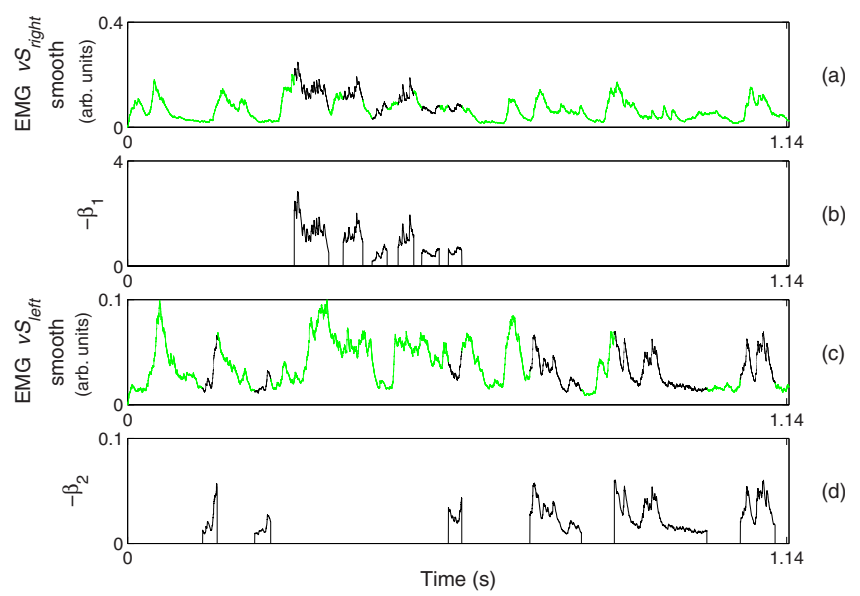

FIG. 4. (Color online) Extraction of the driving parameters of the model from the smoothed EMG activities of the $v S$ muscles. Rectification of the EMG signal and smoothing by low-pass filtering of the (a) right and (c) left $v S$ activities. In both panels, phonation time and $\alpha=\alpha_{\text {on }}$ are indicated with a black line; also nophonation and $\alpha=\alpha_{\text {off }}$ are indicated with a green (light gray) line. The driving parameter $\beta_{i}^{\text {proposed }}$ is shown at times where $\alpha=\alpha_{\text {on }}$ in panels (b) and (d).

formed with a circuit that rectifies the activity and smoothes it by low-pass filtering it. The circuit integrates the following equation:

$$
\frac{d v S}{d t}=-\tau v S+|v S|
$$

with $\tau=10 \mathrm{~ms}$ and $|v S|$ the rectified value of the activity of the ventral muscle. The output of the circuit is shown in panels a and c of Fig. 4. In order to select the optimal set of physiological signals to drive the model, we plotted the smoothed $v S$ signal (from now on $v S$ activity) as a function of pitch. It is natural to select the activity of the right $v S$ muscle in order to generate part of the plot since it has been established that only the right side is responsible for highfrequency sounds [12]. Therefore, for vocalizations of fundamental frequencies above $1.8 \mathrm{kHz}$, we plotted the right $v S$ activity as a function of pitch. The way of treating lowfrequency sounds is more subtle, since both sides are active during at least part of them. We selected left $v S$ activity to drive the model when synthesizing low-frequency sounds. This choice was made after an inspection of the plots of left and right activities as functions of the pitch. In the left panel of Fig. 5, we display, for the high-frequency sounds, the right activity as a function of the pitch and for the low-frequency sounds, the left activity as a function of the pitch. In the right panel of Fig. 5, we plotted the right activity as a function of the pitch for all syllables. Notice that in the right panel, there are levels of the activity leading to two plausible pitches. This ambiguity suggests additional phenomena might be taking place, such as the activity of muscles not yet measured or a nonlinear effect introduced by a coupling between the sources. The only combination that defines a bijective relationship is when left activity is used for low-frequency sounds and right activity for high-frequency sounds, thus
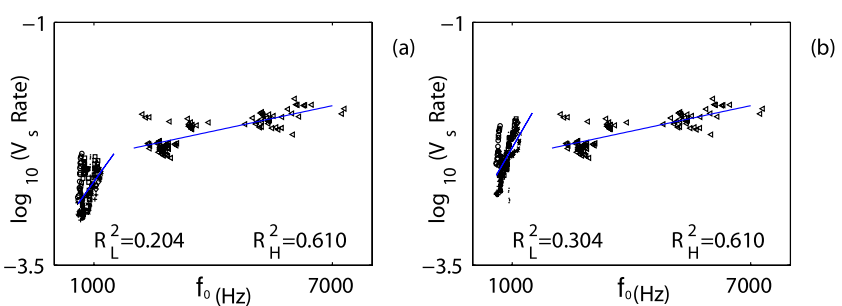

FIG. 5. (Color online) Activity as a function of the pitch, $f_{0}$. In the left panel (a), the pitch of the low-frequency syllables $\left(f_{0}\right.$ $<1.8 \mathrm{kHz}$ ) was plotted as a function of the left-side ventral muscle activity. In (b), the pitch of those syllables was plotted as a function of the right ventral activity. In both panels, the pitch of the highfrequency syllables was plotted as a function of the right ventral activity.

making it the optimal choice in order to drive the model.

In order to synthesize song by driving the model with physiological recordings, we need to find a transformation from the recorded EMG activities to the model parameters $(\alpha, \beta)$ that were previously computed. As before, $\alpha$ is taken as one of two values that place the model out or in an oscillatory regime. We measured air sac pressure by inserting a cannula into a thoracic air sac, with the other end connected to a pressure transducer (see [11]). Air sac pressure larger than a threshold value and sound level above average ambient noise were the conditions for setting $\alpha=\alpha_{\text {on }}$. The parameters $\beta^{\text {proposed }}$ are responsible for the frequency modulation and spectral content of the synthesized sounds. We proposed

$$
\beta_{i}^{\text {proposed }}=a_{0, i}+a_{1, i} v S_{i}(t)+a_{2, i} v S_{i}^{2}(t)
$$

for relating ventral muscle activity with the parameters $\beta^{\text {proposed }}$ in the model. We chose $a_{j, i}$ in order to minimize $\chi^{2}=\chi^{2}\left(\beta_{i}^{\text {proposed }}-\beta_{i}^{\text {numeric }}\right)$, with $i=1,2$ corresponding to high-frequency or low-frequency regimes for $\beta$ and to right or left for $v S$ recordings, respectively. The time parameters $\beta_{i}^{\text {numeric }}$ refer to the time-dependent parameters computed above. In our example, the values obtained are $\left(a_{0,1}, a_{1,1}, a_{2,1}\right)=(-6697,152.65,0.0848)$ and $\left(a_{0,2}, a_{1,2}, a_{2,2}\right)$ $=(0,-17.79,0.0016)$. These parameters where fit using only three $\beta_{i}^{\text {proposed }}$ and $\beta_{i}^{\text {numeric }}$ values at times surrounding segment II in Fig. 2 for high frequency and for low frequency using two values at times surrounding segment I. Notice that since we used only a few points within two segments of the data to fit the parameters and then reproduced the whole song, our fitted model has predictive power. $\beta_{i}^{\text {proposed }}$ at times where $\alpha=\alpha_{\text {on }}$ are shown in panels b and d of Fig. 4. With this fit, we are capable of physiologically driving a device integrating the equations of the model.

We implemented our model on a DSP mounted on a Texas Instruments TMS320C6713 DSK board. It consists of a set of analog input-output channels and a DSP operating at 225 $\mathrm{MHz}$. A program was written in $\mathrm{C}$ that makes use of the sampling capabilities of the board to digitize data corresponding to the physiological activity recordings, perform a numerical integration of the model, and convert the output to an analog signal (the synthesized song).

In order to perform a real-time integration of the model of 
the vocal organ, we used these transformations to define the parameters to feed the DSP. The board digitized the data at a $48 \mathrm{kHz}$ sampling rate. Within each sampling step, the processor integrated the equations accounting for the labial motion. Forward Euler's method was chosen due to its low computational cost, which allowed for 18 iterations to be carried out between samples. Two independent integrations of the model were performed. Each numerically solved variable, $x_{1,2}$, was converted to an analog signal by the DSP at the same rate the input was digitized. The solutions were used to generate a sound signal, whose spectrogram is displayed in Fig. 2 (bottom). Beyond the qualitative similarity, we quantified the agreement between the natural song and the one synthesized with the DSP using the same procedure as the one described above. For the pitch, the mean distance was $\left\langle d_{\text {pitch }}\right\rangle=0.223, \mathrm{STD}=0.296$, while for the SCI $\left\langle d_{\mathrm{SCI}}\right\rangle$ $=0.281, \mathrm{STD}=0.252$.

In this work, we built an electronic device which is capable of translating physiological instructions into song in real time. We did it by programming a DSP so that the device can read the experimental data, integrate the dynamical equations of a physical model for the problem, and use these solutions to synthesize song.

The real-time generation of high-frequency sounds with specific spectral contents presents a challenge. We show that the state of technology can overcome it. The use of digital technology is a qualitative improvement on previous biomimetic vocal solutions [12]: the control of the system's param- eters either for improving the model or as a source of altered feedback is flexible and precise.

DSP technology is being implemented in a variety of biologically inspired problems and is likely to become a standard solution for a variety of biomimetic applications $[13,14]$. In particular, brain machine interfaces (BMIs) typically read data and perform statistical analysis leading to the reconstruction of the parameters that can be used to drive peripheral devices. In this work, we use DSP technology to build a biomimetic solution by asking the device to integrate the equations modeling the physics behind the problem. We propose that this strategy severely reduces the computational demand, thus enhancing the capabilities of current technology for biomimetical applications.

In particular, a well-functioning subject-controlled vocal prosthesis can lay the foundation for similar devices in humans and thus provide directly health-related data and procedures. In this framework, the DSP technology can incrementally improve the quality of synthesized vocal production by incorporating progressive understanding of the physical processes and relevant physiological details involved.

This work was financially supported by University of Buenos Aires, National Institute on Deafness and Other Communications Disorders Grants No. R01 DC-04390 and No. R01 DC-06876, Santa Fe Institute, and Consejo Nacional de Investigaciones Científicas y Técnicas.
[1] H. J. Chiel and R. D. Beer, Trends Neurosci. 20, 553 (1997).

[2] J. D. Sitt, A. Amador, F. Goller, and G. B. Mindlin, Phys. Rev. E 78, 011905 (2008).

[3] A. Amador and G. B. Mindlin, Chaos 18, 043123 (2008).

[4] S. H. Strogatz, Nonlinear Dynamics and Chaos: With Applications to Physics, Biology, Chemistry, and Engineering (Perseus Books, Reading, Massachussetts 2001).

[5] I. R. Titze and D. W. Martin, J. Acoust. Soc. Am. 104, 1148 (1998).

[6] T. Gardner, G. Cecchi, M. Magnasco, R. Laje, and G. B. Mindlin, Phys. Rev. Lett. 87, 208101 (2001).

[7] D. K. Arrowsmith, An Introduction to Dynamical Systems (Cambridge University Press, London, 1990).
[8] E. M. Arneodo and G. B. Mindlin, Phys. Rev. E 79, 061921 (2009).

[9] J. Perry and G. A. Bekey, CRC Crit. Rev. Bioeng. 7, 1 (1981).

[10] G. B. Mindlin, T. J. Gardner, F. Goller, and R. Suthers, Phys. Rev. E 68, 041908 (2003).

[11] F. Goller and B. G. Cooper, Ann. N.Y. Acad. Sci. 1016, 130 (2004)

[12] D. Zysman, J. M. Mendez, B. Pando, J. Aliaga, F. Goller, and G. B. Mindlin, Phys. Rev. E 72, 051926 (2005).

[13] A. B. Schwartz, X. Tracy Cui, D. J. Weber, and D. W. Moran, Neuron 52, 205 (2006).

[14] S. Darmanjian et al., IEEE Engineering in Medicine and Biology Society 1, 2502 (2006). 